\title{
Article \\ The Temporal Dynamics of Rumen Microbiota in Early Weaned Lambs
}

\author{
Shiqin Wang ${ }^{1,2,+} \mathbb{D}$, Jianmin Chai ${ }^{1,3,+} \mathbb{D}$, Guohong Zhao ${ }^{1,2}$, Naifeng Zhang ${ }^{1} \mathbb{D}$, Kai Cui ${ }^{1}$, Yanliang Bi ${ }^{1}$, \\ Tao Ma ${ }^{1}(\mathbb{D})$, Yan Tu ${ }^{1}\left(\mathbb{D}\right.$ and Qiyu Diao ${ }^{1, *(\mathbb{D})}$
}

1 Key Laboratory of Feed Biotechnology of the Ministry of Agriculture, Institute of Feed Research of Chinese Academy of Agricultural Sciences, Beijing 100081, China; wshq1988@163.com (S.W.); jchai@uark.edu (J.C.); zhaoguoh@foxmail.com (G.Z.); zhangnaifeng@caas.cn (N.Z.); cuikai@caas.cn (K.C.); vetbi2008@163.com (Y.B.); matao@caas.cn (T.M.); tuyan@caas.cn (Y.T.)

2 Anhui Province Key Laboratory of Animal Nutritional Regulation and Health, College of Animal Science, Anhui Science and Technology University, Chuzhou 233100, China

3 Department of Animal Science, Division of Agriculture, University of Arkansas, Fayetteville, AR 72701, USA

* Correspondence: diaoqiyu@caas.cn; Tel.: +86-010-8210-6055

+ These authors contributed equally to this work.

check for updates

Citation: Wang, S.; Chai, J.; Zhao, G.; Zhang, N.; Cui, K.; Bi, Y.; Ma, T.; Tu, Y.; Diao, Q. The Temporal Dynamics of Rumen Microbiota in Early Weaned Lambs. Microorganisms 2022, 10, 144. https://doi.org/10.3390/ microorganisms10010144 Academic Editor: Benoit St Pierre

Received: 30 November 2021 Accepted: 8 January 2022

Published: 11 January 2022

Publisher's Note: MDPI stays neutral with regard to jurisdictional claims in published maps and institutional affiliations.

Copyright: (C) 2022 by the authors. Licensee MDPI, Basel, Switzerland. This article is an open access article distributed under the terms and conditions of the Creative Commons Attribution (CC BY) license (https:// creativecommons.org/licenses/by/ $4.0 /)$.

\begin{abstract}
Weaning affects the development of ruminal bacteria in lambs during early life. However, the temporal dynamics of rumen microbiota in early weaned lambs is unknown compared to conventionally weaned lambs. In this study, one group was reared with their dams (control, $\mathrm{CON}$ ) and conventionally weaned at 49 days (d), while the other lambs were weaned at $21 \mathrm{~d}$ (early weaning, EW) using starter. Rumen microbial samples collected at 26, 35, and $63 \mathrm{~d}$ were used for next-generation sequencing. Here, we found that the abundance and diversity of rumen microbiota in EW were significantly lower at 26 and $35 \mathrm{~d}$ than the CON. Linear discriminant analysis Effect Size (LEfSe) analysis was performed to identify the signature microbiota for EW at these three ages. At 26 d, Prevotella 7, Syntrophococcus, Sharpea, Dialister, Pseudoscardovia, and Megasphaera in the rumen of the EW group had greater relative abundances. At $35 \mathrm{~d}$, the Lachnospiraceae_NK3A20_group was enriched in CON. On 63 d, Erysipelotrichaceae_UCG-002 was abundant in EW. Syntrophococcus and Megaspheaera in EW lambs were abundant at 26 and $35 \mathrm{~d}$, but kept similar to CON at $63 \mathrm{~d}$. The relative abundance of Erysipelotrichaceae_UCG-002 at all-time points was consistently higher in the EW group. In conclusion, early weaning led to a significant decrease in rumen microbiota richness and diversity in the short term. The changes in rumen microbiota are associated with the persistence of weaning stress. The temporal dynamics of relative abundances of Syntrophococcus, Megasphaera, and Ruminococcaceae_UCG-014 reflect the weaning stress over a short period and rumen recovery after early weaning.
\end{abstract}

Keywords: lamb; early weaning; rumen microbiota; solid diet; rumen development; rumen maturation; weaning stress

\section{Introduction}

Rumen, as a vital organ, plays a critical role in livestock. The structure and function of the rumen develops rapidly in the early stages of life, such as non-rumination, transition, and rumination. Previous studies have shown that early weaning can promote the early development of rumen structure and function [1,2]. For young ruminants, rumen maturation and its microbiota evolution are directly related to health and performance. Many factors influence microbial colonization, such as the dam's vaginal microbiome and the types of microbes in the surrounding environment [3-5]. Feeding modes also affect the direct transmission of bacteria from the mother and the environment to newborns [6]. As rumen develops, its microbial community changes. Moreover, factors including weaning, feeding strategies, solid feed, starter composition, etc. can affect the early establishment 
and maturation of ruminal microbiota of lambs [2,7-10]. Weaning is one of the most stressful events in the life of a newborn. Commercial farms often separate ewes and lambs before natural weaning. The early separation results in the breaking of the ewe-lamb bond, with changes occurring in both the physical and social environment at the same time, combining also with the end of suckling and the complete replacement of milk by solid food. The latest research shows that weaning age affects the development of the ruminal bacteria in lambs during early life [2]. Other research has shown that weaning significantly influences the morphological and functional development of the rumen, and bacterial community composition [11].

Since there is currently no standard for early weaning, the effects of early weaning on microbiota are controversial, but it plays a key role in animal performance and development. Until now, there have been two ways of conducting early weaning, including using liquid milk replacement and direct feeding starter. In a previous study, lambs could be weaned at as early as $10 \mathrm{~d}$ using a liquid milk replacer [12]. Using a starter to wean lambs is practical in many studies $[11,13]$. The different weaning strategies result in different levels of animal performance and profit. However, there is no doubt that early weaning will cause a drop in feed consumption and a decrease in the growth rate of lambs in a short period [14,15], and lambs can recover after several weeks [16]. Previous research suggests that early-life nutritional intervention determines the initial rumen microbial community, but the persistence of the effects in later life is weak [17]. Therefore, it has been suggested that alterations of the microbiota for optimizing rumen function may be the most effective approach for rumen development during or immediately following the weaning transition [18]. However, the longitudinal changes in rumen microbiota caused by early weaning are currently unclear.

Based on our previous study, early weaning of lambs at $21 \mathrm{~d}$ (early weaning, EW) reduced the average daily gain and affected rumen fermentation parameters in the short term after early weaning compared to conventional weaning (CON) at $49 \mathrm{~d}$ when all lambs had the same weaning starter regime [14]. Here, we continue to explore the effects of early weaning using starter on rumen microbiome of lambs weaned at either 21 or 49 days of age. We hypothesized that the composition of rumen microbiota in early weaned lambs is different in the short and long-term. This study could provide a perspective for further understanding of early microbial colonization rules for young ruminants.

\section{Materials and Methods}

\subsection{Experimental Design, Animal Management, and Sampling}

The study was conducted from October to December 2018, at Runlin sheep farm $\left(\mathrm{N} 36^{\circ} 82^{\prime}, \mathrm{E} 115^{\circ} 83^{\prime}\right)$ in Liaocheng city, Shandong province, China. The experiment protocol was approved by the Animal Ethics and Humane Animal Care of the Chinese Academy of Agricultural Sciences (protocol\#: FRI-CAAS-20180810).

A total of 60 neonatal Hu lambs (48 males and 12 females) with similar birth weights $(3.82 \pm 0.46 \mathrm{~kg})$ were selected and they were born from double pregnancy. Lambs were randomly assigned into two groups based on body weight at 21 days of age. One group was reared with their dams and conventionally weaned (control, CON) at $49 \mathrm{~d}$, while the lambs in the other group were separated from their dams at $21 \mathrm{~d}$ (early weaning, EW). This trial lasted from 21 to $63 \mathrm{~d}$ for both CON and EW groups.

From their birthday to $21 \mathrm{~d}$, all lambs $(n=60)$ were reared with ewes $(n=12)$ in 6 well ventilated sheep pens $(4 \mathrm{~m} \times 5 \mathrm{~m})$ with controlled temperature and humidity. Each pen had ewes $(n=5)$ and their lambs ( $n=10,8$ male and 2 female). From $21 \mathrm{~d}$ to $63 \mathrm{~d}$, control lambs with their ewe stayed in the same pen, while ewes of EW group were removed and EW lambs were kept in the same pen. All lambs consumed the same starter ( $21.5 \%$ crude protein, and $15.1 \% \mathrm{NDF}$ ) from 7 to $35 \mathrm{~d}$, while another starter $(21.5 \%$ crude protein, and $18.9 \% \mathrm{NDF}$ ) was provided from 36 to $63 \mathrm{~d}$. All lambs had free access to starter and water during the whole experiment. 
Six male lambs per treatment (two lambs from each pen) were randomly selected and sacrificed at 26,35 , and $63 \mathrm{~d}$. The rumen digesta were collected and aliquoted to two tubes. One of tubes was snap-frozen in liquid nitrogen and subsequently stored at $-80^{\circ} \mathrm{C}$ freezer for further microbial analyses. Another one was stored at $-20^{\circ} \mathrm{C}$ for analyses of the volatile fatty acids (VFAs) and ammonia nitrogen $\left(\mathrm{NH}_{3}-\mathrm{N}\right)$ as described previously [14].

\subsection{DNA Extraction and $16 S$ rRNA Sequencing}

After samples were thawed, the DNA of the rumen content samples was extracted. The extraction procedure followed the instructions of the E.Z.N.A. Stool DNA Kit (Omega Biotek, Norcross, GA, USA). After completed the DNA extraction, 1\% agarose gel electrophoresis and spectrophotometry and a Thermo NanoDrop 2000 UV microphotometer (Thermo Scientific, Waltham, MA, USA) were used to detect DNA quality and concentration. Then, we used bacterial DNA as a template, and primers with specific barcode to perform bacterial $16 \mathrm{~S}$ rDNA gene amplification in the V3-V4 sequencing region. The steps of bacterial 16S rRNA gene sequence amplification are as follows: PCR started with an initial denaturing step at $94{ }^{\circ} \mathrm{C}$ for $5 \mathrm{~min}$, followed by 28 cycles at $94{ }^{\circ} \mathrm{C}$ for $30 \mathrm{~s}, 55^{\circ} \mathrm{C}$ for $30 \mathrm{~s}$, and $72{ }^{\circ} \mathrm{C}$ for $60 \mathrm{~s}$, and a final extension at $72{ }^{\circ} \mathrm{C}$ for $7 \mathrm{~min}$. The PCR products were detected using $2 \%$ agarose gel electrophoresis. During DNA extraction and PCR step, negative and positive controls were included for quality control. We did not detect any PCR products from these two negative controls on an agarose gel. Therefore, we believe that contamination was minimal and unlikely to have affected biological variation in the different groups. TruSeq ${ }^{\circledR}$ DNA PCR-Free DNA Library Construction Kit (Illumina Inc., San Diego, CA, USA) was used for library construction [19]. After the constructed library was quantified by the Qubit Fluorometer (Thermo Fisher Scientific, Waltham, MA, USA) and KAPA Illumina Library Quantification Kits (Roche, Indianapolis, IN, USA), it was sequenced on the Illumina MiSeq-PE300 platform (Illumina Inc., San Diego, CA, USA) and generated $2 \times 300$ bp paired-end reads.

\subsection{Bioinformatics and Data Analysis}

After original sequencing data was obtained, Trimmomatic (v0.36) (SLIDINGWINDOW:50:20 and MINLEN:120) was used to trim adapters and filter sequences, producing sequences with a phred score over 20 and a length over $120 \mathrm{bp}$. Then, Pear (v0.9.6) were used to remove the reads with $\mathrm{N}$ base exceeding $5 \%$. The chimeras and singletons were detected and removed by Vsearch software (v2.7.1) and high-quality sequences were clustered into operational taxonomic units (OTUs) at a 97\% similarity level using the Uparse algorithm in QIIME (Version 1.8.0) [20]. The most frequently occurring sequence in OTUs was selected as the representative sequence of OTUs, and aligned against to GreenGenes bacterial database using the RDP Classifier (Version 2.2) Bayesian algorithm. The microbial sequencing data of this study are available in the NCBI SRA database with the BioProject ID: PRJNA792702.

Samples were normalized to 24,773 sequencing reads, and Qiime software (v.1.8.0) [21] was used for statistical analysis of alpha diversity, including observed-species and Shannon index. The Unifrac distances (both unweighted and weighted) were calculated based on the difference in evolution information between each sample sequence in Qiime software, and $\mathrm{R}$ packages were used for visualization of principal co-ordinates analysis (PCoA) [22]. ANOSIM was performed to analyze the differences in community structure between groups.

Linear discriminant analysis Effect Size (LEfSe) analysis was performed to determine the genera differentiated in their relative abundances between the three age groups [23]. Taxa with an LDA Score $>2.5$ were considered as exhibiting a significant effect size. To find the relationship between rumen microbiota and fermentation parameters, Spearman correlation analysis was performed. A heat map was used to show the correlations. 


\section{Results}

\subsection{Diversity and Richness of Rumen Microbial Communities}

A total of 1,705,188 high-quality sequences were obtained from 36 rumen samples with an average of 47,366 sequences per sample, and 2820 operational taxonomic units (OTUs) were detected based on $97 \%$ similarity. With a subsample of 24,733 sequences (the minimum number of reads detected per sample) for each sample, the Good's coverage $(>0.98)$ revealed that our data provided sufficient sequencing depth to accurately describe the rumen bacterial composition of the lambs used in this study.

Compared with the CON group, the abundance and diversity of rumen microbiota in the EW group was significantly lower at 26 and $35 \mathrm{~d}(p<0.05)$ as indicated by both Shannon index and the number of observed species, while the differences between the two groups at $63 \mathrm{~d}$ were not statistically significant (Figure 1A,B). Then, changes of alpha diversities of rumen microbiota in lambs of different ages were also analyzed. For the CON group, the alpha diversities of the rumen microbiota were significantly reduced $(p<0.05)$ from 26 to $35 \mathrm{~d}$, and increased $(p<0.05)$ from 35 to $63 \mathrm{~d}$ (Figure S1). Notably, the number of observed species of the CON groups at $63 \mathrm{~d}$ was lower $(p<0.05)$ than at $26 \mathrm{~d}$, although no difference of Shannon index was observed between these two ages. Regarding the EW groups, while no differences of both Shannon Index and the number of observed species were detected between 26 and $35 \mathrm{~d}$, significant increases of alpha diversities at $63 \mathrm{~d}$ were observed.

A
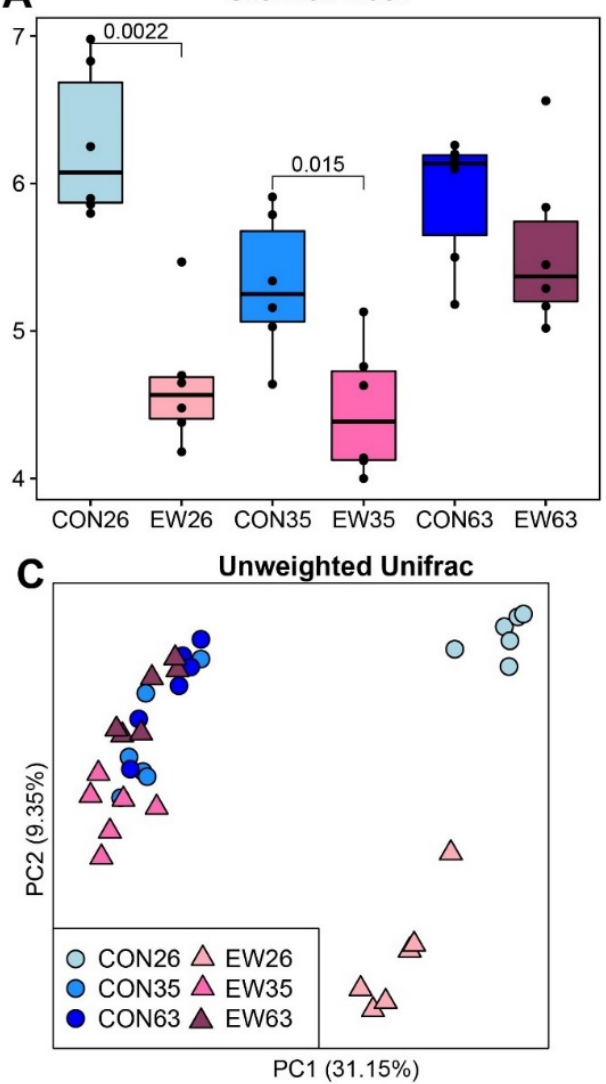

B

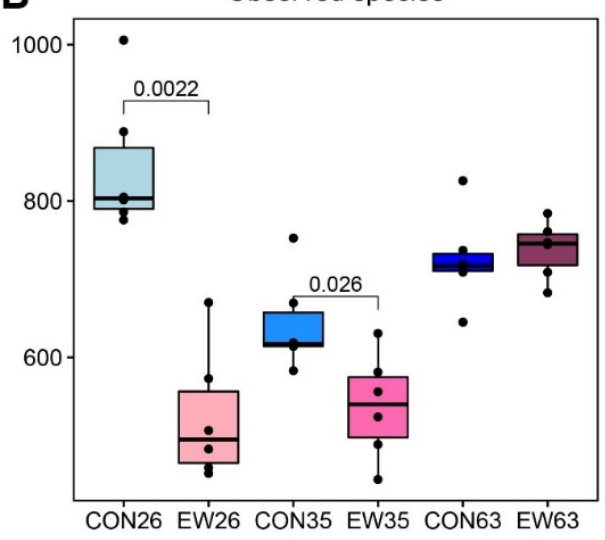

D

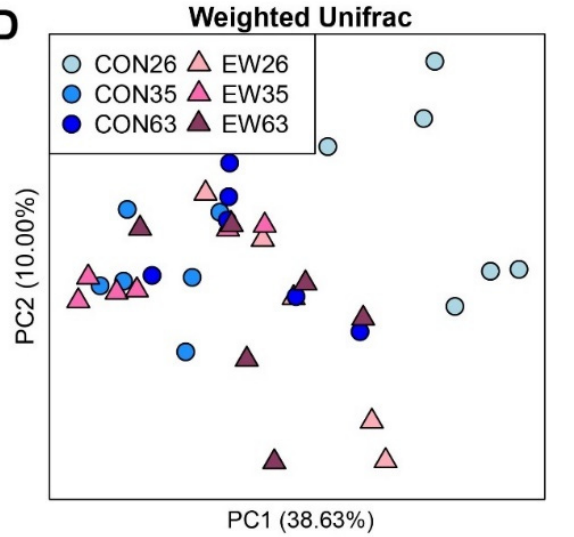

Figure 1. Alpha diversity and similarity of rumen microbiota in control $(\mathrm{CON})$ and early weaned (EW) lambs at 26, 35, and 63 days of age (d). (A,B): Alpha diversity in the rumen microbial community based on the Shannon index and observed species; (C,D): The principal coordinate analysis (PCoA) based on the unweighted and weighted Unifrac distances. Each point represents a unique sample. CON26 = control at $26 \mathrm{~d}$; EW = early weaning group at $26 \mathrm{~d}$, the rest can be deduced by analogy.

Principal Coordinate Analysis (PCoA) was performed based on unweighted and weighted Unifrac distances (Figure 1C,D). The rumen microbiota of lambs in the EW and 
CON groups showed distinct clusters on $26 \mathrm{~d}$ (ANOSIM, $\mathrm{R}=0.88, p=0.003$ ) based on unweighted Unifrac distance, and EW and CON were also distinct at 35 and $63 \mathrm{~d}$ (ANOSIM: $\mathrm{R}=0.15, p=0.08 ; \mathrm{R}=0.32, p=0.03$ ). Based on Weighted Unifrac distance, these patterns were also observed, although increased variation within the group. Moreover, age effects on bacterial community structure were observed. For instance, the microbiota of both CON and EW groups at $26 \mathrm{~d}$ were distinct compared to other ages.

\subsection{Bacterial Composition at Different Taxonomical Levels}

A total of 16 phyla of the rumen contents in both CON and EW groups were identified. The main phyla across all samples were Firmicutes with the highest relative abundance, followed by Bacteroidetes, Actinobacteria, and Proteobacteria, accounting for over $95 \%$ of total reads (Figure 2A). At $26 \mathrm{~d}$, the relative abundance of Firmicutes in the CON group was $34.8 \%$, while its relative abundance in the EW group increased to $50.8 \%$. At the same time, the relative abundance of Bacteroides in EW was 39.3\% compared to its relative abundance in CON with 52.3\%. Additionally, Firmicutes in both CON and EW groups increased from 26 to $35 \mathrm{~d}$, and subsequently decreased at $63 \mathrm{~d}$. However, the opposite pattern of Bacteroides with age was observed.
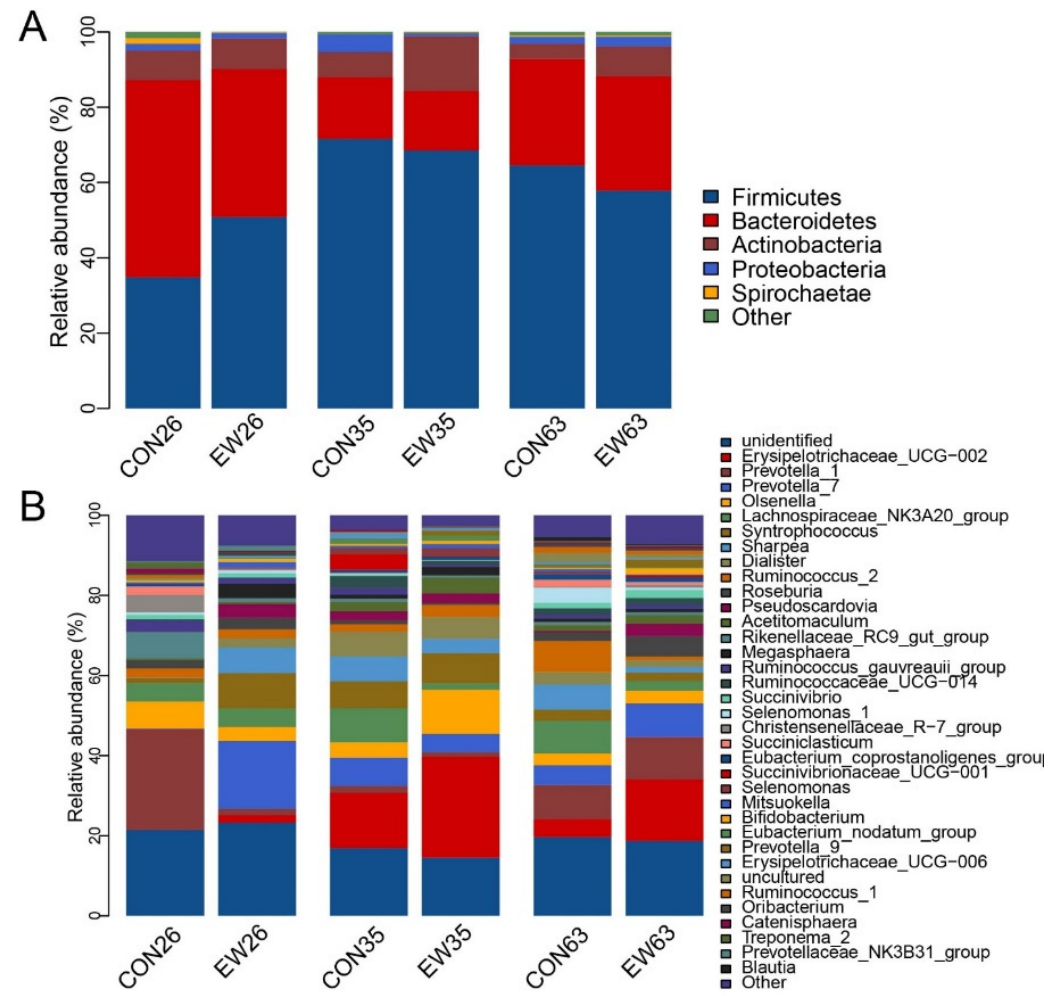

Figure 2. The composition of rumen microbiome at phylum and genus level. (A): The composition of rumen microbiome at phylum level; (B): The composition of major rumen genera. CON26 = control at $26 \mathrm{~d}$; EW = early weaning group at $26 \mathrm{~d}$. The rest can be deduced by analogy.

At the genus level, a total of 208 genera were identified in the rumen contents of all lambs. The top 18 genera had relative abundances more than $1.0 \%$, accounting for $66.74 \%$ in total. These bacteria mainly included Erysipelotrichaceae UCG-002, Prevotella 7, Prevotella 1, Olsenella, Lachnospiraceae NK3A20 group, Sharpea, and Syntrophococcus, etc. (Figure 2B). Then, we analyzed the ruminal bacteria composition of lambs affected by early weaning at different ages. At 26 d, these genera of Prevotella 7, Syntrophococcus, Sharpea, Dialister, Pseudoscardovia, and Megasphaera in the EW group had greater $(p<0.05)$ relative abundances, while the relative abundances of Prevotella 1, Rikenellaceae_RC9_gut_group and Ruminococcaceae_UCG-014 were higher $(p<0.05)$ in the CON group. On $35 \mathrm{~d}$, the relative abundance of the Lachnospiraceae_NK3A20_group in the EW group was reduced significantly $(p<0.05)$ 
compared with the CON group. At $63 \mathrm{~d}$, the relative abundance of Ruminococcus_2 in the rumen of EW group was significantly lower $(p<0.05)$, but Erysipelotrichaceae_UCG-002 increased significantly when compared to CON group (Table S1). Additionally, the relative abundance of Erysipelotrichaceae_UCG-002 reached its peak at 35 d, while Prevotella 1 had the lowest relative abundance at $35 \mathrm{~d}$ in the lambs in the EW group.

\subsection{Bacterial Biomarkers for Early Weaning (EW) at Different Ages}

To identify the bacterial taxa differentiating CON and EW groups at different ages, Linear discriminant analysis Effect Size (LEfSe) analysis was performed at the genus level. At $26 \mathrm{~d}$, the bacteria with significant differences in the CON group were Prevotella 1, Rikenellaceae RC9 gut group, and Christensenellaceae R 7 group, while the bacterial biomarkers for the EW group were Prevotella 7, Syntrophococcus, Sharpea, Megaspheaera, Pseudoscardovia, and Erysipelotrichaceae UCG-002 (Figure 3A). Upon sampling rumen microbiota at $35 \mathrm{~d}$, Lachnospiraceae_NK3A20_group was enriched in the CON group, but Erysipelotrichaceae UCG-002 was identified as biomarker for the EW group (Figure 3B). At the end of this trial (63 d), rumen microbiota in lambs of the CON group had high relative abundances of Ruminococcus 2 and Lachnospiraceae_NK3A20_group, whereas Erysipelotrichaceae UCG-002 and Eubacterium ruminantium group were over-represented in the EW group (Figure 3C).
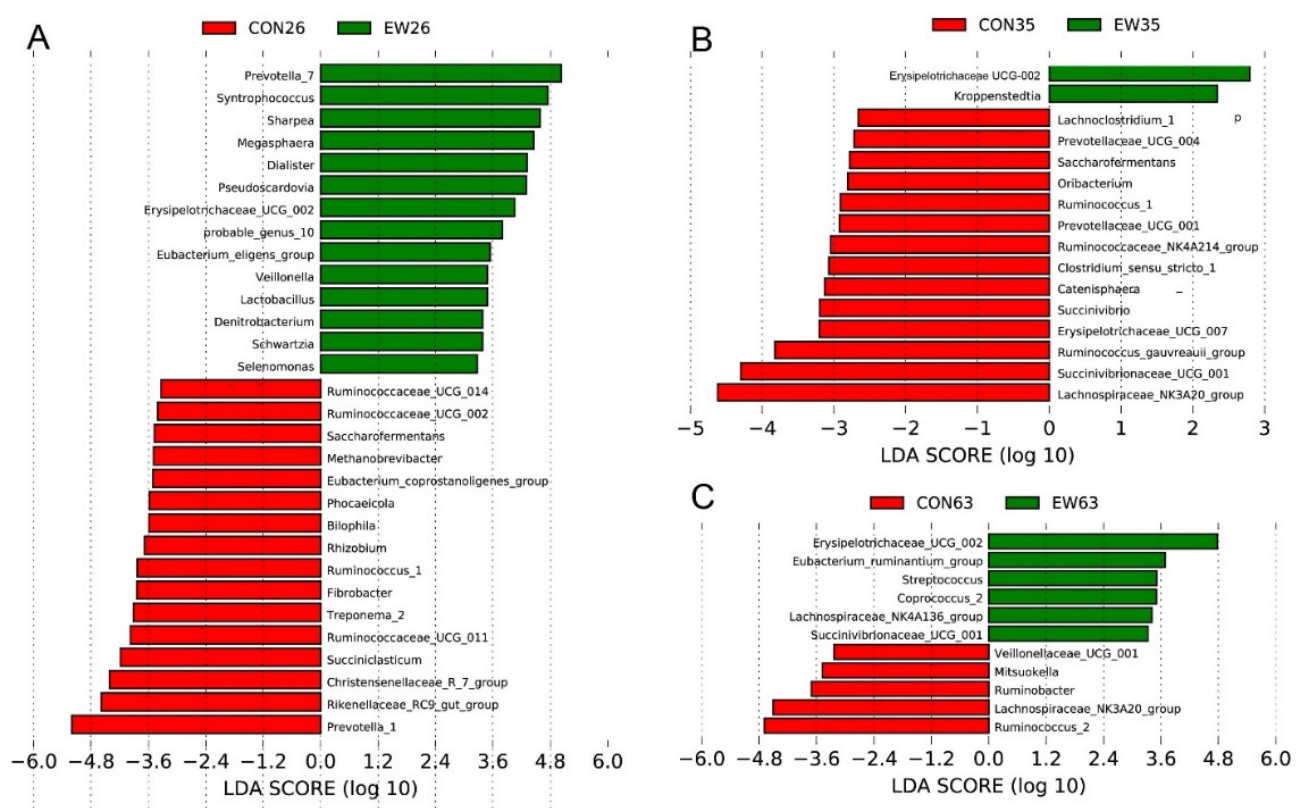

Figure 3. LEfSe analyses of rumen microbiota. LEfSe identified significantly different bacteria at the genus level as differentiating the two groups (control (CON) and early weaned (EW)) at $26 \mathrm{~d}(\mathrm{~A})$, $35 \mathrm{~d}(\mathbf{B})$ and $63 \mathrm{~d}(\mathbf{C})$. Genera in this graph were statistically significant $(p<0.05)$ and had an LDA Score $>2.5$, which was considered a significant effect size.

\subsection{Temporal Dynamics of Rumen Microbiota in CON and EW Groups}

To better understand how early weaning affects the maturation process of rumen microbiota in early weaned lambs, it is necessary to compare the temporal dynamics of control groups at the same ages. Regarding the longitudinal changes of rumen microbiota in CON groups from 26 to $63 \mathrm{~d}$, LEfSe algorithm was performed to find the biomarkers for each age (Figure S2A). For example, Prevotella 1, Christensenellaceae R 7 group, and Erysipelotrichaceae_UCG-004 were higher at 26 d; Prevotella 7, Erysipelotrichaceae_UCG-002, Dialister, Pseudoscardovia, and Ruminococcaceae_UCG-014 were enriched at $35 \mathrm{~d}$; and Sharpea, Megaspheaera, and Erysipelotrichaceae UCG-009 were abundant at 63 d. For rumen microbiome of early weaning lambs, bacterial biomarkers for each age were also identified. At 26 d, Syntrophococcus, Megaspheaera, Selenomonas_1 and Erysipelotrichaceae_UCG-004 as the 
EW signatures were also classified. At 35 d, Erysipelotrichaceae UCG-002, Acetitomaculum, and Selenomonas were enriched in the ruminal community. At $63 \mathrm{~d}$, the abundant genera included Prevotella 1, Ruminococcaceae_UCG-014, Roseburia, Succinivibrio, Prevotella 9, Syntrophococcus, and Christensenellaceae $\mathrm{R} 7$ group.

To further understand that the bacterial biomarkers for both CON and EW groups changed with ages, the corresponding genera were selected to create a better visualization. For Syntrophococcus and Megasphaera identified as EW biomarkers, their relative abundances in the CON and EW groups had different patterns changed with ages (Figure 4A,B). In other words, the differences of their relative abundances between $\mathrm{CON}$ and EW deceased with age, which is consistent with the results of diversities. Ruminococcaceae_UCG-014 were higher in the CON compared to the EW group, and its relative abundance was higher during 35 and 63 d (Figure 4C). Erysipelotrichaceae UCG-002 was consistently higher in EW group from 26 to $63 \mathrm{~d}$ and its relative abundances in both CON and EW groups increased with ages (Figure $4 \mathrm{D})$.
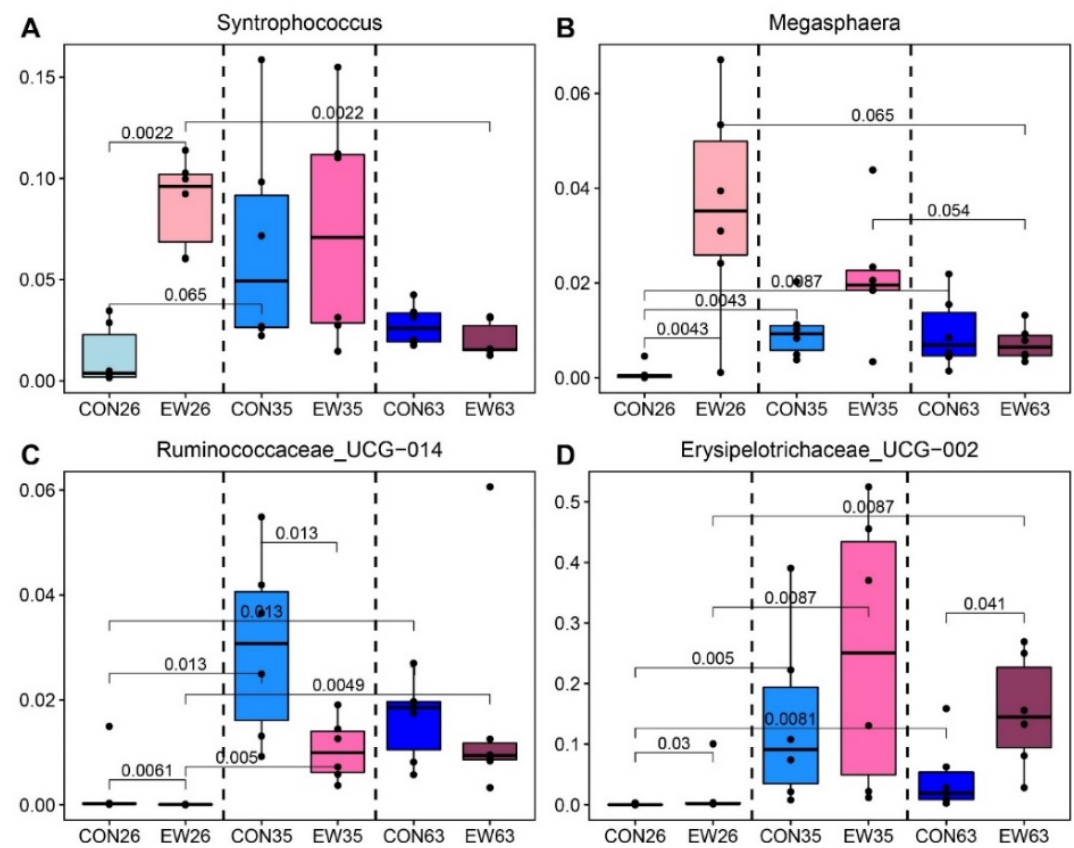

Figure 4. Temporal dynamics of the bacterial biomarkers for lambs changed with ages. The important bacterial biomarkers, including Syntrophococcus (A), Megasphaera (B), Ruminococcaceae_UCG-014 (C) and Erysipelotrichaceae UCG-002 (D), showed different patterns of temporal dynamics in control $(\mathrm{CON})$ and early weaned (EW) groups. CON26 = control at $26 \mathrm{~d} ; \mathrm{EW}=$ early weaning group at $26 \mathrm{~d}$. The rest can be deduced by analogy.

\subsection{Rumen Bacteria Associated with Fermentation Parameters}

The data of rumen fermentation parameters were obtained from our previous study [14]. Briefly, the concentration of Total VFA, molar proportion of acetate and propionate in the EW group increased at 5 days after early weaning. A number of significant correlations were found between the rumen microbiota and fermentation parameters (Figure 5). For example, rumen ammonia concentration was positively correlated with the relative abundances of Erysipelotrichaceae_UCG-002, Acetitomaculum, and Ruminococcaceae_UCG-014; acetate had a positive correlation with Pseudoscardovia; propionate was negatively correlated with the Rikenellaceae_RC9_gut_group but positively correlated with Megasphaera and Sharpea; butyrate was negatively correlated with Pseudoscardovia and Megasphaera. 


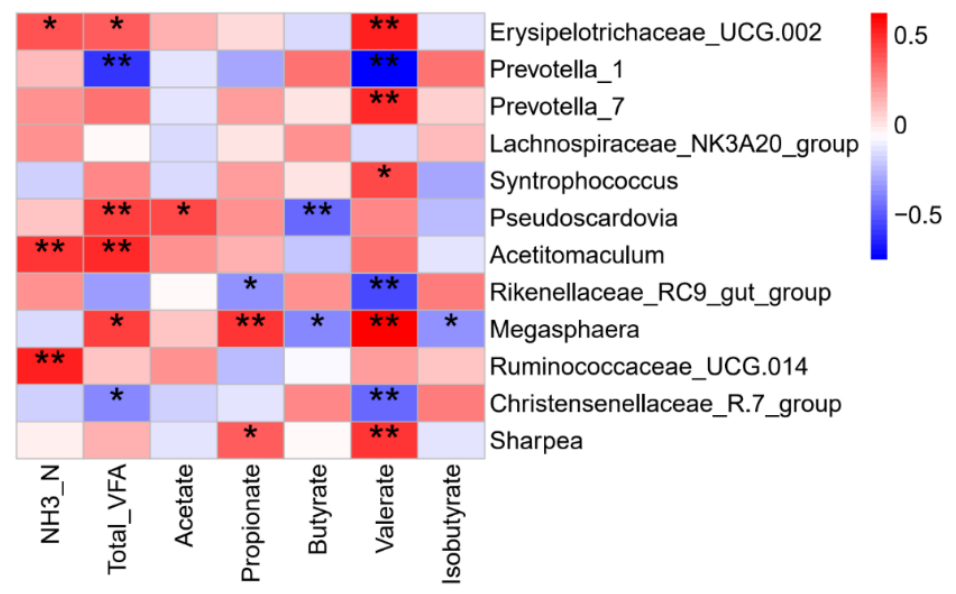

Figure 5. Spearman correlation analysis of ruminal microbiota and rumen fermentation parameters. Color represents the correlation coefficient, with red representing a positive correlation and blue denoting a negative correlation. ${ }^{*} p<0.05$ and ${ }^{* *} p<0.01$.

\section{Discussion}

\subsection{The Diversity and Richness of Rumen Microbiota Influenced by Early Weaning}

The diversity and richness index are usually used to evaluate the stability of an ecosystem. Our results show that early weaning caused the decreased richness and diversity of rumen microbiota of lambs, and its impacts mainly happened on 5 and 14 days after weaning at $21 \mathrm{~d}$. At the same time, we observed a significant difference in beta diversity of the rumen microbiota between the EW and CON lambs on $26 \mathrm{~d}$. The dissimilarity of the structure of the rumen microbiota between the two groups decreased on 42 days after early weaning. These results suggest that early weaning changed the process of colonization of the rumen microbiota of lambs in a short period, while the diversity and structure of rumen microbial communities could recover at 42 days after weaning. These results partially differ from previous research which found that the alpha diversity and beta diversity between weaning and post-weaning for lambs receiving the strategy of artificial feeding with milk replacer and weaning at $30 \mathrm{~d}$ were not significantly different when compared to controls [2]. Another study of the same feeding strategy showed that alpha diversity was not significantly influenced between pre- and post-weaning whether or not lambs were weaned at 21 or $35 \mathrm{~d}$ [13]. Differences with reported results may be related to different feeding strategies. One of the studies pointed out that supplementary feeding with alfalfa before weaning could avoid changes in the rumen microbial diversity and abundance before and after weaning [8]. Feeding mode is an important driver of early microbial colonization, influencing the structure and function of lambs' gut microbiome [6]. Our study is based on the feeding mode of breastfeeding, which may cause higher stress in a short period. However, at 42 days after early weaning, the rumen microbiome in early weaned lambs could reach the same level as control lambs. This suggests that the mode of weaning lambs at 21 days of age and feeding with solid feed may be feasible for actual production.

After the birth of young ruminants, the rumen microbiota is in a process of constant change with age and the maturation of rumen [24,25]. So, we further studied the diversity and richness changes with age of the rumen microbiota of lambs in the two groups. In the CON group, the diversity and richness of bacterial communities increased with age, which is in accordance with the results of previous studies [7,11,25]. This is mainly related to the continuous changes in lambs' breast milk and starter intake to continuously adjust and adapt to rumen fermentation substrates. At $63 \mathrm{~d}$, the diversity and abundance of rumen microorganisms increased, indicating that the rumen microbiota was established more completely to better adapt to the digestion of solid feed. In EW group, the rumen microbial diversity and richness of lambs at $63 \mathrm{~d}$ were higher than at 26 and $35 \mathrm{~d}$, which 
is consistent with the previous research reported [2,11]. As we know, for early weaned lambs, the diet was directly converted from breast milk to solid feed and lambs' feed intake of starer after weaning increased sharply. Previous studies showed that the feeding of starter is an important factor affecting the colonization of young ruminants before ruminating $[8,9,26]$. The above results indicate that early nutritional intervention could affect the initial microbial formation in the rumen, and the influence of weaning on the rumen community is the most obvious [27]. Early weaning stress has adverse effects on lambs' growth and health [14], and impacts the richness and diversity of rumen microbiota. So, it is necessary to know the short-term and long-term effects of early weaning stress on the rumen microbiota of lambs, and how the rumen microbiotas change during the stress transition and recovery periods.

\subsection{The Compositions of Rumen Microbiota Are Associated with Early Weaning}

At the phylum level, the rumen microbiota of lambs in control and early weaning groups are mainly Firmicutes, Bacteroidetes, Actinobacteria, and Proteobacteria, which is consistent with previous research results in lambs $[8,13,24]$. This study shows that early weaning affected the composition of microbes in the rumen of lambs in the short-term. As in the phylum, after early weaning, the relative abundance of Firmicutes in the rumen significantly increased, while the percent of Bacteroides significantly decreased on day 26 of age. After early weaning, solid feed was increased significantly. The increases in relative abundance of Firmicutes are related to high grain feeding, energy harvesting or feed efficiency [28-30]. Studies on dairy cows have shown that high grain feeding increased the relative abundance of Firmicutes [30]. However, one study showed no significant difference in relative abundance of any of the top four bacterial phyla between different ages [2] and this may be related to different feeding strategies or weaning age. Our previous research found that there were significant differences between the two different feeding strategies of ewe-lamb breastfeeding or artificial feeding [6]. This study was based on the production mode of lambs in most farms where the lambs are breastfed until final weaning.

To further understand the effects of early weaning on rumen microbiota, the signature genera for two groups were identified. Our results show that early weaning mainly influenced the microbial composition at 5 days after weaning. At $26 \mathrm{~d}$ (5 days after early weaning), the composition of the microbiota changed significantly. The relative abundance of the genera, such as Prevotella 7, Sharpea, Syntrophococcus, Dialister, and Megasphaera, increased significantly in early weaned lambs. As we know, Prevotella is one of the dominant genera within phylum Bacteroidetes in the rumen, which is consistent with the results on lambs [31]. It can degrade and utilize starch and plant cell wall polysaccharides, such as xylan, and pectin, in the rumen for fermentation, and the products are acetic acid, succinic acid, and propionic acid [32]. Prevotella also could utilize lactic acid and convert it into propionic acid [33]. Thus, an increase in the relative abundance of Prevotella is beneficial for ruminants that consume a high concentrate diet [34,35]. Syntrophococcus, Dialister, Megasphaera, and Sharpea are all members of Firmicutes that could digest various carbohydrates to produce short chain fatty acids [36]. The increased relative abundances of these bacteria result in the improved ability to digest carbohydrates with increasing solid feed intake in the early weaned lambs after early weaning. An increase of Dialister has been associated with hyposalivation [37], which may play a role in altering the buffering capacity of the rumen and fluid turnover. Sharpea is the lactate-producer [38], and Megasphaera can converse the lactate to butyrate [39]. Thus, Sharpea was accompanied by a corresponding increase in the percentage of Megasphaera, which increased butyrate production. Overall, the increased relative abundance of these genera in the rumen after weaning can promote digestion and utilization of carbohydrates. After early weaning, we found a significant decrease in the relative abundance of Rikenellaceae_RC9_gut_group and Ruminococcaceae_UCG-014. The family Rikenellaceae is found to be involved in the degradation of structural carbohydrates $[40,41]$. One study reported that cows fed high-starch diets with supplemental oil decreased the relative abundance of Rikenellaceae_RC9 [41]. 
During the recovery transition period (14 days after early weaning), only a small number of bacteria were affected after early weaning. Erysipelotrichaceae UCG-002 and Selenomonas_1 were enriched in the ruminal community of EW lambs. Compared with the previous stage, the biggest change was in Erysipelotrichaceae UCG-002, which became the dominant bacteria, which was associated with the rumen total volatile fatty acids concentration. Selenomonas_1 is a non-spore forming anaerobic bacteria that plays an essential role in the production of volatile fatty acids, especially propionic acid in the rumen [42]. At this stage, weaning stress decreased after the adaptation of starter intake, as we observed that the starter intake increased significantly, and the rumen developed rapidly in our previous study [14].

On day 42 of age after early weaning, the differences between CON and EW groups became smaller. Erysipelotrichaceae_UCG-002 was still the dominant bacteria in the early weaning group, while Rumenococcus 2 was the dominant bacteria in the control group. At this stage, all lambs were weaned and fed the same solid feed, resulting in a similar concentration of rumen fermented substrates. When challenged by a microbial community different to the rumen conditions of the host animal, the rumen can successfully rebuild its own microbiota [43]. This indicated that a time-dependent effect on some bacteria before stabilization may occur with age. The persistent changes of the same bacterial species in the rumen are the result of adapting to the composition of carbohydrates in the transition from liquid feed to a starter.

Similar research results also show that lambs weaned at 21 or $35 \mathrm{~d}$ did not affect the rumen microbiota and rumen fermentation parameters at $50 \mathrm{~d}$ [13]. The difference in rumen microbiota of young animals is not affected by weaning time or weaning method, but is mainly related to whether weaning or not. One study on calves has shown that weaning had a significant impact on the composition and structure of the rumen microbiota, while weaning methods have less impacts on it [44]. Maternal separation at weaning immediately shifted the composition of the gut microbiota in all animals, revealing fitness differences among species. It is likely that these differences are linked to maternal separation-associated stress. Similarly, O'Mahoney et al. reported that feces of adult mice that had undergone maternal separation for $3 \mathrm{~h}$ per day from postnatal days 2-12 presented an altered microbiota composition when compared with the nonseparated control animals [45]. However, the impacts of milk withdrawal on rumen microbiota composition at weaning are less probable and still need further study.

\subsection{The Temporal Dynamics of Rumen Microbiota after Early Weaning}

Compared with weaning, there are higher numbers of bacterial genera which changed with ages. Thus, the temporal dynamics of the bacterial biomarkers for early weaned lambs were investigated, which could help us understand the persistent effects of early weaning. Relative abundance of Syntrophococcus was lower in EW lambs at $26 \mathrm{~d}$, but retained similar relative abundance with controls at other ages. Syntrophococcus is involved in the utilization of non-fibrous carbohydrates and produces acetic acid [46]. After early weaning, the percent of Syntrophococcus increased, which is similar to the results of the recent study on calves [47]. The relative abundance of Syntrophococcus decreased at $63 \mathrm{~d}$ in both groups compared to $35 \mathrm{~d}$, which may be due to ages and diet. Megasphaera is a lactic acid-utilizing bacterium. The proportion of Megasphaera in the early weaning group is higher than that of the control group at 26 and $35 \mathrm{~d}$, which proves its relationship with early weaning and starter intake. Research showed that Megasphaera is an ecologically important rumen bacterium that metabolizes lactate and relieves rumen acidosis induced by a high-grain-diet [48]. Our results showed that Megasphaera was positively correlated with the concentration of propionate, valerate, and total VFA while negatively correlated with butyrate, which reflects the importance of Megasphaera. Previous studies on lambs showed that starter feeding promoted the increase of the relative abundance of Megasphaera and would be beneficial for weaning of starter-fed lambs $[49,50]$. After early weaning, lambs only consume solid feed with high grains provide conditions for Megasphaera growth, while its relative abundance became 
similar to controls due to disappearance of the weaning stress. Ruminococcaceae_UCG-014 changed with age in the CON compared to EW group. Ruminococcaceae is an important fibrolytic bacteria in the guts of mammals [51]. One study has shown that increasing the level of fiber in the diet will lead to Ruminococcaceae_UCG-014 growth [52]. In the current study, the relative abundance of Ruminococcaceae_UCG-014 increased with increasing age. Moreover, Ruminococcaceae_UCG-014 were positively correlated with rumen ammonia concentration. This shows that the ability of lambs to digest fiber and protein enhanced with age. The percent of Erysipelotrichaceae_UCG_002 also increased after early weaning, especially in early weaned rumen, and was positively correlated with the concentration of total VFA and valerate. Erysipelotrichaceae_UCG_002 belongs to the family of Erysipelotrichaceae of Firmicutes member which was previously reported to be associated with VFA synthesis [53]. Therefore, Erysipelotrichaceae may promote cholesterol production and accumulation [54], which suggests it may be related to increased starter intake after early weaning. Unfortunately, the function of Erysipelotrichaceae_UCG_002 is still elusive and thus requires an in-depth exploration in the future.

\section{Conclusions}

Early weaning could cause a significant decrease in richness and diversity in rumen microbiota in the short-term, and lead to corresponding shifts in the composition of rumen microbiota. At 42 days after early weaning, its effects on rumen microbial composition decline. The temporal dynamics of relative abundances of Syntrophococcus, Megasphaera and Ruminococcaceae_UCG-014 reflect the weaning stress over a short period and rumen recovery after early weaning. However, the persistent effects of early weaning may have impacts on the maturated rumen considering the higher relative abundances of Erysipelotrichaceae_UCG_002 in early weaned lambs at the end of this trial. Overall, this study demonstrates the temporal dynamics of rumen microbiota in early weaned lambs, which allow us to better understand the effects of early weaning.

Supplementary Materials: The following are available online at https:/ /www.mdpi.com/article/ 10.3390/microorganisms10010144/s1, Table S1: Differences in the composition of rumen microbial taxon of lambs among different; Figure S1: Alpha diversities of rumen microbiota in lambs; Figure S2: The bacterial biomarker identified by LEfSe for each age in CON and EW groups.

Author Contributions: S.W. and J.C. contributed to animal trials, sample collection, data analysis and primary drafting. Q.D. and N.Z. contributed to the experimental design and project management. G.Z., K.C., Y.B. and T.M. helped to sample collection and analysis. Y.T. and Q.D. contribute to reviewing and proofing. All authors have read and agreed to the published version of the manuscript.

Funding: This work was supported by the China Agriculture Research System of MOF and MARA (CARS-38), Cooperative Guidance Project of "Prospering Inner Mongolia through Science and Technology" in 2021(2021CG0024), National Natural Science Foundation of China (32172764) and grants from National Key R\&D Program Projects (2018YFD0501902).

Institutional Review Board Statement: The study was approved by the Institutional Review Board of Animal Ethics and Humane Animal Care of the Chinese Academy of Agricultural Sciences (protocol\#: FRI-CAAS-20180810).

Informed Consent Statement: Not applicable.

Data Availability Statement: All data are contained within the article or Supplementary Materials.

Acknowledgments: We thank students in the ruminant nutrition laboratory of Feed Research Institute of Chinese Academy of Agricultural Sciences (CAAS) for their technical assistance. We thank Linqing Runlin Sheep Industry Co. Ltd. (Shandong Province, China) for their assistance with the animal experiment.

Conflicts of Interest: The authors declare no conflict of interest. 


\section{References}

1. Carballo, O.C.; Khan, M.A.; Knol, F.W.; Lewis, S.J.; Stevens, D.R.; Laven, R.A.; McCoard, S.A. Impact of weaning age on rumen development in artificially reared lambs. J. Anim. Sci. 2019, 97, 3498-3510. [CrossRef] [PubMed]

2. Mao, H.; Zhang, Y.; Yun, Y.; Ji, W.; Jin, Z.; Wang, C.; Yu, Z. Weaning age affects the development of the ruminal bacterial and archaeal community in hu lambs during early life. Front. Microbiol. 2021, 12, 636865. [CrossRef] [PubMed]

3. Klein-Jobstl, D.; Quijada, N.M.; Dzieciol, M.; Feldbacher, B.; Wagner, M.; Drillich, M.; Schmitz-Esser, S.; Mann, E. Microbiota of newborn calves and their mothers reveals possible transfer routes for newborn calves' gastrointestinal microbiota. PLoS ONE 2019, 14, e0220554. [CrossRef] [PubMed]

4. $\quad$ Owens, C.E.; Huffard, H.G.; Nin-Velez, A.I.; Duncan, J.; Teets, C.L.; Daniels, K.M.; Ealy, A.D.; James, R.E.; Knowlton, K.F.; Cockrum, R.R. Microbiomes of various maternal body systems are predictive of calf digestive bacterial ecology. Animals 2021, 11, 2210. [CrossRef]

5. Arshad, M.A.; Hassan, F.U.; Rehman, M.S.; Huws, S.A.; Cheng, Y.; Din, A.U. Gut microbiome colonization and development in neonatal ruminants: Strategies, prospects, and opportunities. Anim. Nutr. 2021, 7, 883-895. [CrossRef]

6. Bi, Y.; Cox, M.S.; Zhang, F.; Suen, G.; Zhang, N.; Tu, Y.; Diao, Q. Feeding modes shape the acquisition and structure of the initial gut microbiota in newborn lambs. Environ. Microbiol. 2019, 21, 2333-2346. [CrossRef]

7. Zhuang, Y.; Chai, J.; Cui, K.; Bi, Y.; Diao, Q.; Huang, W.; Usdrowski, H.; Zhang, N. Longitudinal investigation of the gut microbiota in goat kids from birth to postweaning. Microorganisms 2020, 8, 1111. [CrossRef]

8. Yang, B.; Le, J.; Wu, P.; Liu, J.; Guan, L.L.; Wang, J. Alfalfa intervention alters rumen microbial community development in hu lambs during early life. Front. Microbiol. 2018, 9, 574. [CrossRef]

9. Lv, X.; Chai, J.; Diao, Q.; Huang, W.; Zhuang, Y.; Zhang, N. The signature microbiota drive rumen function shifts in goat kids introduced to solid diet regimes. Microorganisms 2019, 7, 516. [CrossRef]

10. Lv, F.; Wang, X.; Pang, X.; Liu, G. Effects of supplementary feeding on the rumen morphology and bacterial diversity in lambs. Peer.J. 2020, 8, e9353. [CrossRef]

11. Li, C.; Zhang, Q.; Wang, G.; Niu, X.; Wang, W.; Li, F.; Li, F.; Zhang, Z. The functional development of the rumen is influenced by weaning and associated with ruminal microbiota in lambs. Anim. Biotechnol. 2020, 1-17. [CrossRef] [PubMed]

12. Chai, J.M.; Ma, T.; Wang, H.C.; Qi, M.L.; Tu, Y.; Diao, Q.Y.; Zhang, N.F. Effect of early weaning age on growth performance, nutrient digestibility, and serum parameters of lambs. Anim. Prod. Sci. 2017, 57, 110-115. [CrossRef]

13. Zhang, Q.; Li, C.; Niu, X.; Zhang, Z.; Li, F.; Li, F. The effects of milk replacer allowance and weaning age on the performance, nutrients digestibility, and ruminal microbiota communities of lambs. Anim. Feed. Sci. Technol. 2019, 257, 114263. [CrossRef]

14. Wang, S.; Ma, T.; Zhao, G.; Zhang, N.; Tu, Y.; Li, F.; Cui, K.; Bi, Y.; Ding, H.; Diao, Q. Effect of age and weaning on growth performance, rumen fermentation, and serum parameters in lambs fed starter with limited ewe-lamb interaction. Animals 2019, 9, 825. [CrossRef]

15. McCoard, S.A.; Cristobal-Carballo, O.; Knol, F.W.; Heiser, A.; Khan, M.A.; Hennes, N.; Johnstone, P.; Lewis, S.; Stevens, D.R. Impact of early weaning on small intestine, metabolic, immune and endocrine system development, growth and body composition in artificially reared lambs. J. Anim. Sci. 2020, 98, skz356. [CrossRef]

16. Chai, J.; Diao, Q.; Wang, H.; Tu, Y.; Tao, X.; Zhang, N. Effects of weaning age on growth, nutrient digestibility and metabolism, and serum parameters in Hu lambs. Anim. Nutr. 2015, 1, 344-348. [CrossRef]

17. Belanche, A.; Yanez-Ruiz, D.R.; Detheridge, A.P.; Griffith, G.W.; Kingston-Smith, A.H.; Newbold, C.J. Maternal versus artificial rearing shapes the rumen microbiome having minor long-term physiological implications. Environ. Microbiol. 2019, 21, 4360-4377. [CrossRef]

18. Dill-McFarland, K.A.; Breaker, J.D.; Suen, G. Microbial succession in the gastrointestinal tract of dairy cows from 2 weeks to first lactation. Sci. Rep. 2017, 7, 40864. [CrossRef]

19. Fomenky, B.E.; Chiquette, J.; Bissonnette, N.; Talbot, G.; Chouinard, P.Y.; Ibeagha-Awemu, E.M. Impact of Saccharomyces cerevisiae boulardii cncmi-1079 and Lactobacillus acidophilus Bt1386 on total Lactobacilli population in the gastrointestinal tract and colon histomorphology of holstein dairy calves. Anim. Feed. Sci. Technol. 2017, 234, 151-161. [CrossRef]

20. Edgar, R.C. Uparse: Highly accurate otu sequences from microbial amplicon reads. Nat. Methods 2013, 10, 996-998. [CrossRef]

21. Schloss, P.D.; Gevers, D.; Westcott, S.L. Reducing the effects of PCR amplification and sequencing artifacts on 16s rRNA-based studies. PLoS ONE 2011, 6, e27310. [CrossRef]

22. Wang, Y.; Sheng, H.F.; He, Y.; Wu, J.Y.; Jiang, Y.X.; Tam, N.F.; Zhou, H.W. Comparison of the levels of bacterial diversity in freshwater, intertidal wetland, and marine sediments by using millions of illumina tags. Appl. Environ. Microbiol. 2012, 78, 8264-8271. [CrossRef] [PubMed]

23. Segata, N.; Izard, J.; Waldron, L.; Gevers, D.; Miropolsky, L.; Garrett, W.S.; Huttenhower, C. Metagenomic biomarker discovery and explanation. Genome Biol. 2011, 12, R60. [CrossRef]

24. Wang, Y.; Zhang, H.; Zhu, L.; Xu, Y.; Liu, N.; Sun, X.; Hu, L.; Huang, H.; Wei, K.; Zhu, R. Dynamic distribution of gut microbiota in goats at different ages and health states. Front. Microbiol. 2018, 9, 2509. [CrossRef] [PubMed]

25. Jami, E.; Israel, A.; Kotser, A.; Mizrahi, I. Exploring the bovine rumen bacterial community from birth to adulthood. ISME J. 2013, 7, 1069-1079. [CrossRef]

26. Chai, J.; Lv, X.; Diao, Q.; Usdrowski, H.; Zhuang, Y.; Huang, W.; Cui, K.; Zhang, N. Solid diet manipulates rumen epithelial microbiota and its interactions with host transcriptomic in young ruminants. Environ. Microbiol. 2021, 23, 6557-6568. [CrossRef] 
27. Dill-McFarland, K.A.; Weimer, P.J.; Breaker, J.D.; Suen, G. Diet influences early microbiota development in dairy calves without long-term impacts on milk production. Appled Environ. Microbiol. 2019, 85, e02141-18. [CrossRef]

28. Jami, E.; White, B.A.; Mizrahi, I. Potential role of the bovine rumen microbiome in modulating milk composition and feed efficiency. PLoS ONE 2014, 9, e85423. [CrossRef]

29. Myer, P.R.; Smith, T.P.; Wells, J.E.; Kuehn, L.A.; Freetly, H.C. Rumen microbiome from steers differing in feed efficiency. PLoS ONE 2015, 10, e0129174.

30. Pan, X.; Xue, F.; Nan, X.; Tang, Z.; Wang, K.; Beckers, Y.; Jiang, L.; Xiong, B. Illumina sequencing approach to characterize thiamine metabolism related bacteria and the impacts of thiamine supplementation on ruminal microbiota in dairy cows fed high-grain diets. Front. Microbiol. 2017, 8, 1818. [CrossRef] [PubMed]

31. Zhang, Q.; Li, C.; Niu, X.; Zhang, Z.; Li, F.; Li, F. An intensive milk replacer feeding program benefits immune response and intestinal microbiota of lambs during weaning. BMC Vet. Res. 2018, 14, 366. [CrossRef]

32. Stevenson, D.M.; Weimer, P.J. Dominance of prevotella and low abundance of classical ruminal bacterial species in the bovine rumen revealed by relative quantification real-time PCR. Appl. Microbiol. Biotechnol. 2007, 75, 165-174. [CrossRef]

33. Belanche, A.; Doreau, M.; Edwards, J.E.; Moorby, J.M.; Pinloche, E.; Newbold, C.J. Shifts in the rumen microbiota due to the type of carbohydrate and level of protein ingested by dairy cattle are associated with changes in rumen fermentation. J. Nutr. 2012, 142, 1684-1692. [CrossRef] [PubMed]

34. Thomas, M.; Webb, M.; Ghimire, S.; Blair, A.; Olson, K.; Fenske, G.J.; Fonder, A.T.; Christopher-Hennings, J.; Brake, D.; Scaria, J. Metagenomic characterization of the effect of feed additives on the gut microbiome and antibiotic resistome of feedlot cattle. Sci. Rep. 2017, 7, 12257. [CrossRef] [PubMed]

35. Ran, T.; Jiao, P.; AlZahal, O.; Xie, X.; Beauchemin, K.A.; Niu, D.; Yang, W. Fecal bacterial community of finishing beef steers fed ruminally protected and non-protected active dried yeast. J. Anim. Sci. 2020, 98, skaa058. [CrossRef] [PubMed]

36. Rey, M.; Enjalbert, F.; Combes, S.; Cauquil, L.; Bouchez, O.; Monteils, V. Establishment of ruminal bacterial community in dairy calves from birth to weaning is sequential. J. Appl. Microbiol. 2014, 116, 245-257. [CrossRef] [PubMed]

37. Hayashi, Y.; Saito, T.; Ohshima, T.; Nakagawa, Y.; Arita, T.; Yashima, A.; Makino, T.; Konnai, R.; Gomi, K.; Arai, T.; et al. Terminal Rflp analysis to determine the oral microbiota with hyposalivation. Arch. Microbiol. 2014, 196, 489-496. [CrossRef] [PubMed]

38. Kamke, J.; Kittelmann, S.; Soni, P.; Li, Y.; Tavendale, M.; Ganesh, S.; Janssen, P.H.; Shi, W.; Froula, J.; Rubin, E.M.; et al. Rumen metagenome and metatranscriptome analyses of low methane yield sheep reveals a sharpea-enriched microbiome characterised by lactic acid formation and utilisation. Microbiome 2016, 4, 56. [CrossRef]

39. Mackie, R.I.; Gilchrist, F.M. Changes in lactate-producing and lactate-utilizing bacteria in relation to $\mathrm{pH}$ in the rumen of sheep during stepwise adaptation to a high-concentrate diet. Appl. Environ. Microbiol. 1979, 38, 422-430. [CrossRef]

40. Qiu, Q.; Gao, C.; Gao, Z.; Rahman, M.A.U.; He, Y.; Cao, B.; Su, H. Temporal dynamics in rumen bacterial community composition of finishing steers during an adaptation period of three months. Microorganisms 2019, 7, 410. [CrossRef]

41. Zened, A.; Combes, S.; Cauquil, L.; Mariette, J.; Klopp, C.; Bouchez, O.; Troegeler-Meynadier, A.; Enjalbert, F. Microbial ecology of the rumen evaluated by $454 \mathrm{Gs}$ Flx pyrosequencing is affected by starch and oil supplementation of diets. FEMS Microbiol. Ecol. 2013, 83, 504-514. [CrossRef]

42. Scheifinger, C.C.; Linehan, B.; Wolin, M.J. H2 production by selenomonas ruminantium in the absence and presence of methanogenic bacteria. Appl. Microbiol. 1975, 29, 480-483. [CrossRef]

43. Weimer, P.J.; Stevenson, D.M.; Mantovani, H.C.; Man, S.L. Host specificity of the ruminal bacterial community in the dairy cow following near-total exchange of ruminal contents. J. Dairy Sci. 2010, 93, 5902-5912. [CrossRef] [PubMed]

44. Meale, S.J.; Li, S.; Azevedo, P.; Derakhshani, H.; Plaizier, J.C.; Khafipour, E.; Steele, M.A. Development of ruminal and fecal microbiomes are affected by weaning but not weaning strategy in dairy calves. Front. Microbiol. 2016, 7, 582. [CrossRef]

45. O'Mahony, S.M.; Marchesi, J.R.; Scully, P.; Codling, C.; Ceolho, A.M.; Quigley, E.M.; Cryan, J.F.; Dinan, T.G. Early life stress alters behavior, immunity, and microbiota in rats: Implications for irritable bowel syndrome and psychiatric illnesses. Biol. Psychiatry 2009, 65, 263-267. [CrossRef] [PubMed]

46. Cui, Z.; Wu, S.; Liu, S.; Sun, L.; Feng, Y.; Cao, Y.; Chai, S.; Zhang, G.; Yao, J. From maternal grazing to barn feeding during pre-weaning period: Altered gastrointestinal microbiota contributes to change the development and function of the rumen and intestine of yak calves. Front. Microbiol. 2020, 11, 485. [CrossRef] [PubMed]

47. Hao, Y.; Guo, C.; Gong, Y.; Sun, X.; Wang, W.; Wang, Y.; Yang, H.; Cao, Z.; Li, S. Rumen fermentation, digestive enzyme activity, and bacteria composition between pre-weaning and post-weaning dairy calves. Animals 2021, 11, 2527. [CrossRef]

48. Chen, L.; Shen, Y.; Wang, C.; Ding, L.; Zhao, F.; Wang, M.; Fu, J.; Wang, H. Megasphaera elsdenii lactate degradation pattern shifts in rumen acidosis models. Front. Microbiol. 2019, 10, 162. [CrossRef] [PubMed]

49. Liu, J.; Bian, G.; Sun, D.; Zhu, W.; Mao, S. Starter feeding altered ruminal epithelial bacterial communities and some key immune-related genes' expression before weaning in lambs. J. Anim. Sci. 2017, 95, 910-921. [CrossRef] [PubMed]

50. Lin, L.; Xie, F.; Sun, D.; Liu, J.; Zhu, W.; Mao, S. Ruminal microbiome-host crosstalk stimulates the development of the ruminal epithelium in a lamb model. Microbiome 2019, 7, 83. [CrossRef]

51. Liu, J.; Bian, G.; Sun, D.; Zhu, W.; Mao, S. Starter feeding supplementation alters colonic mucosal bacterial communities and modulates mucosal immune homeostasis in newborn lambs. Front. Microbiol. 2017, 8, 429. [CrossRef] [PubMed]

52. Yu, M.; Gao, T.; Liu, Z.; Diao, X. Effects of dietary supplementation with high fiber (Stevia residue) on the fecal flora of pregnant sows. Animals 2020, 10, 2247. [CrossRef] [PubMed] 
53. Ahmad, A.A.; Yang, C.; Zhang, J.; Kalwar, Q.; Liang, Z.; Li, C.; Du, M.; Yan, P.; Long, R.; Han, J.; et al. Effects of Dietary energy levels on rumen fermentation, microbial diversity, and feed efficiency of yaks (Bos grunniens). Front. Microbiol. 2020, 11, 625. [CrossRef] [PubMed]

54. Goldsmith, J.R.; Sartor, R.B. The role of diet on intestinal microbiota metabolism: Downstream impacts on host immune function and health, and therapeutic implications. J. Gastroenterol. 2014, 49, 785-798. [CrossRef] 